\section{COMPARISON OF CISPLATIN AND MITOMYCIN/5-FU AS RADIOSENSITIZERS IN THE TREATMENT OF VULVAR CANCER - RESULTS OF A SINGLE INSTITUTIONAL COHORT STUDY}

${ }^{1} \mathrm{~V}$ Linz ${ }^{*},{ }^{2} \mathrm{C}$ Schwanbeck, ${ }^{1} \mathrm{~K}$ Anic, ${ }^{3} \mathrm{~J}$ Jäkel, ${ }^{1} \mathrm{MW}$ Schmidt, ${ }^{1} S$ Krajnak, ${ }^{1} \mathrm{R}$ Schwab, ${ }^{1} \mathrm{~W}$ Weikel, ${ }^{1} \mathrm{~A}$ Seeger, ${ }^{1} \mathrm{M}$ Schmidt, ${ }^{2} \mathrm{H}$ Schmidberger, ${ }^{1} \mathrm{~A}$ Hasenburg, ${ }^{1} \mathrm{M}$ Battista. ${ }^{1}$ University Medical Centre of the Johannes Gutenberg University Mainz, Department of Gynaecology and Obstetrics, Mainz, Germany; ${ }^{2}$ University Medical Centre of the Johannes Gutenberg University Mainz, Department of Radiooncology and Radiotherapy, Mainz, Germany; ${ }^{3}$ University Medical Centre of the Johannes Gutenberg University Mainz, Department of Pathology, Mainz, Germany

\subsection{6/ijgc-2021-ESG0.617}

Introduction/Background* Treatment of vulvar cancer contains surgery if applicable and often primary or neo-/adjuvant chemoradiation. Cisplatin and Mitomycin/5-FU are widely used radiosensitizers in vulvar cancer, although evidence is limited. We retrospectively investigated both radiosensitizers for outcome and toxicity.

Methodology We screened the archive for patients treated with chemoradiation for pathologically-confirmed squamous cell cancer of the vulva between 01/2010 - 02/2021 at our institution. The impact of both radiosensitizers on prognosis was compared using Kaplan-Meier method and Cox-Regression analysis.

Result(s)* 127 patients with vulvar cancer were screened. 24 patients received chemoradiation (Cisplatin $n=11$; Mitomycin/ 5 -FU $n=11$; others $n=2$ ) as a neoadjuvant, primary or adjuvant treatment. Median follow-up was 17 months in both groups. Median age was 65 (35-91) years. Patients in the Cisplatin group were older than in the Mitomycin/5-FU group (Median age 73 vs. 55, p=0.007). Median radiation dose in total was $59.6(41.4-85.0)$ Gy. $68.2 \%$ of the patients had FIGO stage III and $18.2 \%$ of the patients had FIGO stage IV before chemoradiation. Both groups showed no differences in terms of FIGO stage.

$72.7 \%$ (8 out of 11) patients receiving Mitomycin/5-FU achieved clinical complete response compared to $45.5 \% \quad(5$ out of 11$)$ receiving Cisplatin $(p=0.309)$. One patient in each group was progressive during chemoradiation. Three patients stopped chemoradiation due to toxicity (Cisplatin $n=2$; Mitomycin/5-FU $\mathrm{n}=1$ ) and one patient in the Cisplatin group died of treatment related sequelae. Radiodermatitis with epitheliolysis were the most common adverse events in both groups. Additionally, four patients in the Mitomycin/5-FU group required treatment for myelotoxicity.

The 2 -year overall survival showed a numerical but not statistically significant difference in favour of the Mitomycin/5FU group $(59.7 \%$ vs. $30.7 \%$; $=0.306)$. The 2-year-recurrence-free-survival was comparable between Mitomycin/5-FU and Cisplatin $(43.6 \%$ vs. $31.8 \%$; $=0.829)$.

Conclusion* Cisplatin and Mitomycin/5-FU showed a numerical but not statistically significant difference in overall survival in vulvar cancer. MitomycinC/5-FU tended to result in a clinical complete response more frequently but required more often a treatment for myelotoxicity. However, overall toxicity of Mitomycin/5-FU was acceptable and may be considered in the treatment of young, healthy patients with locally advanced vulvar cancer.

\section{MANAGEMENT OF SENTINEL NODE PROCEDURE IN VULVAR CANCER IN GERMANY: A SURVEY AMONG GYNECOLOGICAL DEPARTMENTS}

${ }^{1} \mathrm{R}$ Schwab*, ${ }^{1} \mathrm{~T}$ Bührer, ${ }^{1} \mathrm{~J}$ Van der Ven, ${ }^{1} \mathrm{~K}$ Anic, ${ }^{1} \mathrm{~S}$ Krajnak, ${ }^{1} \mathrm{~V}$ Linz, ${ }^{1} \mathrm{~W}$ Weikel, ${ }^{1} \mathrm{M}$ Battista, ${ }^{2} \mathrm{C}$ Dannecker, ${ }^{1} \mathrm{~A}$ Hasenburg. 'University Medical Center Mainz, Department of Gynecology and Obstetrics, Mainz; ${ }^{2}$ University Medical Center Augsburg, Department of Gynecology and Obstetrics, Augsburg, Germany

\subsection{6/ijgc-2021-ESG0.618}

Introduction/Background* Lymph node involvement is the most important prognostic factor for recurrence and survival in vulvar cancer. Sentinel lymphonodectomy (SNLD) successfully replaced inguinofemoral lymphadenectomy (IL) in patients with early vulvar cancer. Nevertheless, management of patients in special situations is not well studied or current data are inconclusive.

Methodology To assess the current management of diagnostic and surgical procedures with respect to SNLD in early vulvar cancer, a web-based survey was mailed to 612 gynecological departments. Data are presented as frequencies of selected items.

Result(s)* A total of 222 hospitals (36.27\%) replied the questionnaire. Only $9.5 \%$ of hospitals did not offer the SNLD and $79.5 \%$ processed the evaluation of sentinel node $(\mathrm{SN})$ by ultrastaging.

After detection of isolated tumor cells (ITCs) or micrometastases, IL was performed in $28.1 \%$ and $60.5 \%$ of hospitals, respectively, and radiation without further surgical intervention was performed in $19.3 \%$ and $23.8 \%$ of hospitals, respectively. In $50.9 \%$ and $15.1 \%$ of cases, hospitals would not initiate any further therapy in patients with ITCs and micrometastases, respectively.

In vulvar cancer of the midline with unilateral localized positive SN, $49.1 \%$ and $48.6 \%$ of hospitals performed ipsilateral or bilateral IF, respectively. Repeat SNLD was performed by $16.2 \%$ of hospitals.

Conclusion* The vast majority of German hospitals are following current guidelines and are offering SNLD in patients with early vulvar cancer. Despite the fact that according to the GROINSS-V-I-study approximately $42 \%$ of positive SN were determined by ultrastaging, only $79.5 \%$ of German hospitals implemented this procedure.

Our findings showed that hospitals would recommend expectant management in patients with ITCs or with micrometastases in $50.9 \%$ and $15.1 \%$ of cases, but current guidelines do not support this approach. Nevertheless, radiation is a good option in these patients, as shown by preliminary results of the GROINSS-V-II-study. Bilateral IL should be performed in patients with unilateral positive $\mathrm{SN}$, as a $\mathrm{SN}$ positivity rate of $22.2 \%$ on the contralateral side was described by a current study and understaging of the groins might be fatal.

Any deviation from current guidelines, as well as applying the SNLD in special situations, should be individually discussed with each patient. 